\title{
FLORES EDÍVEIS COMO RECURSO NATURAL DE COMPOSTOS BIOATIVOS E PROPRIEDADES BIOLÓGICAS
}

\author{
EDIBLE FLOWERS AS A NATURAL RESOURCE OF BIOACTIVE \\ COMPOUNDS AND BIOLOGICAL PROPERTIES
FLORES EDIBLES COMO RECURSO NATURAL DE COMPUESTOS BIOACTIVOS Y PROPIEDADES BIOLÓGICAS

\author{
Sara Vanessa Pinto (27509@ufp.edu.pt)* \\ Adriana Oliveira (26183@ufp.edu.pt)** \\ Carla Sousa (sousasil@ufp.edu.pt)*** \\ M. Conceição Manso (cmanso@ufp.edu.pt)**** \\ Ana F. Vinha (acvinha@ufp.edu.pt)*****
}

\section{RESUMO}

\begin{abstract}
Atualmente, muitas espécies de plantas que produzem flores já fazem parte da alimentação humana, salientando-se em particular as pétalas e sépalas edíveis. Habituais na cozinha desde a Antiguidade, o uso das flores não tem sido usual em Portugal. No entanto, com as novas tendências de recuperar os sabores agridoces e das múltiplas variantes da cozinha de fusão, as flores tornam-se ingredientes muito apreciados. Nem todas as flores são comestíveis. Para além da identificação das mesmas, é importante saber como foram produzidas pois, por exemplo, as flores para decoração ornamental não devem ser utilizadas para consumo humano, uma vez que não têm em consideração as regras de segurança alimentar. Contudo, existe pouca informação sobre algumas espécies de flores destinadas a consumo humano, nomeadamente calêndula (Calendula officinalis L.), camélia (Camellia japonica L.) e rosa (Rosa canina L.). Nesse sentido, o presente trabalho pretendeu contribuir para aumentar o conhecimento neste tema, designadamente ao nível da caracterização química e das propriedades antioxidantes destas espécies botânicas. Embora as três espécies de flores estudadas tenham compostos bioativos e atividade antioxidante, a C. officinalis foi a que apresentou o maior teor em fenólicos totais e carotenoides e, consequentemente, maior atividade antioxidante.
\end{abstract}

Palavras Chave: calendula officinalis I., camellia japonica I., rosa canina I., composição química, atividade antioxidante.

\section{ABSTRACT}

Currently, many plant species that produce flowers are already part of the human diet, in particular edible petals and sepals. Commonl in the kitchen since antiquity, the use of flowers has not been usual in Portugal. However, with new trends in picking up the bittersweet flavours and the multiple variants of fusion cuisine, flowers become highly prized ingredients. It is important to be aware that 
not all flowers are edible. In addition to their identification, it is important to know about their production practices, because if they were not for human consumption the food safety rules may not have been taken into account, such as in the case of ornamental decoration flowers. There is scarce information on some edible flowers species, namely calendula (Calendula officinalis L.), camellia (Camellia japonica L.) and rose (Rosa canina L.). Thus, the present study sets out to contribute to an increased knowledge of the chemical characterization and the antioxidant properties of these botanical species. Although the studied species of flowers had bioactive compounds and antioxidant activity, C. officinalis presented the highest total phenolics and carotenoids content and, consequently, higher antioxidant activity.

Keywords: calendula officinalis I., camellia japonica I., rosa canina l., chemical composition, antioxidant activity.

\section{RESUMEN}

Actualmente, muchas especies de plantas que producen flores ya forman parte de la alimentación humana, en particular, los pétalos y sépalos edibles. Habituales en la cocina desde la Antigüedad, el uso de las flores no ha sido usual en Portugal. Sin embargo, con las nuevas tendencias de recuperar los sabores agrios y las múltiples variantes de la cocina de fusión, las flores se convierten en ingredientes muy apreciados. Es importante tener en cuenta que no todas las flores son comestibles y, además de la identificación de las mismas, es importante saber cómo se produjeron pues, por ejemplo, las flores para decoración ornamental no deben ser utilizadas para el consumo humano, ya que no tienen en consideración las normas de seguridad alimentaria. Sin embargo, hay poca información sobre algunas especies de flores, en particular caléndula (Calendula officinalis L.), camelia (Camellia japonicaL.) y rosa (Rosa caninaL.). En este sentido, el presente trabajo pretendió contribuir a aumentar el conocimiento en este tema, con particularidad a nivel de la caracterización química y de las propiedades antioxidantes de estas especies botánicas. Aunque las tres especies de flores estudiadas tenían compuestos bioactivos y actividad antioxidante, la $C$. officinalis fue la que presentó el mayor contenido en fenólicos totales y carotenoides $y$, consecuentemente, mayor actividad antioxidante.

Palabras clave: calendula officinalis I., camellia japonica I., rosa canina l., composición química, actividad antioxidante.

*Aluna Finalista da Licenciatura em Ciências da Nutrição, da Faculdade de Ciências da Saúde da Universidade Fernando Pessoa, Porto, Portugal.

**Aluna Finalista da Licenciatura em Ciências da Nutrição, da Faculdade de Ciências da Saúde da Universidade Fernando Pessoa, Porto, Portugal.

*** Professora Associada da Faculdade de Ciências da Saúde da Universidade Fernando Pessoa, Porto, Portugal / Investigadora integrada do FP-ENAS ((Unidade de Investigação UFP em Energia, Ambiente e Saúde), CEBIMED (Centro de Estudos em Biomedicina), Universidade Fernando Pessoa), Porto, Portugal.

**** Professora Associada da Faculdade de Ciências da Saúde da Universidade Fernando Pessoa, Porto, Portugal / Investigadora colaboradora do REQUIMTE/LAQV, Departamento de Ciências Químicas, Faculdade de Farmácia, Universidade do Porto, Porto, Portugal / Investigadora integrada do FP-ENAS (Unidade de 
Investigação UFP em Energia, Ambiente e Saúde), Universidade Fernando Pessoa), Porto, Portugal.

***** Professora Auxiliar da Faculdade de Ciências da Saúde da Universidade Fernando Pessoa, Porto, Portugal / Investigadora colaboradora do REQUIMTE/LAQV, Departamento de Ciências Químicas, Faculdade de Farmácia, Universidade do Porto, Porto, Portugal / Investigadora integrada do FP-ENAS ((Unidade de Investigação UFP em Energia, Ambiente e Saúde), CEBIMED (Centro de Estudos em Biomedicina), Universidade Fernando Pessoa), Porto, Portugal.

Submitted: 17th February 2019

Accepted: 11th July 2019 


\section{INTRODUÇÃO}

O crescente interesse em nutracêuticos e alimentos funcionais tem aumentado nos últimos anos, o que se reflete ao nível da investigação da indústria alimentar, que tem como intuito desenvolver novos géneros alimentícios benéficos para a saúde. Assim, muitos estudos têm sido desenvolvidos, nomeadamente, em frutas exóticas e pouco conhecidas (Virgolin et al., 2017; Bailão et al., 2015; Pereira et al., 2013; Rufino et al., 2011), plantas aromáticas (Shukla et al., 2016; Vinha etal., 2015; Kusuma et al., 2014; Ching et al., 2012) e sementes (Montes et al., 2018; Xu et al., 2016; Vinha et al., 2014), realçando o valor nutricional e o teor em compostos bioativos e atividades biológicas, designadamente, o seu potencial antioxidante.

O mercado de flores comestíveis está em expansão, não só nos países industrializados, como no mundo em geral, devido à crescente utilização das mesmas na gastronomia, promovendo o aumento das variedades e do crescimento económico nacional e internacional (Guiné et al., 2017).

Atualmente, as flores comestíveis são utilizadas em preparações culinárias, infusões e/ou bebidas, visando o melhoramento dos atributos sensoriais e promovendo aromas e cores mais atrativas ao consumidor (Pires et al., 2018; Benvenuti et al., 2016). De uma maneira geral, as flores são usadas em molhos, geleias, xaropes, licores, vinagres, mel, óleos, flores cristalizadas, cubos de gelo, saladas, chás e outras bebidas e sobremesas (Tundis et al., 2015; Swithinbank, 2015). Além disso, as flores comestíveis são importantes para a saúde humana devido à sua riqueza em compostos bioativos, sugerindo oportunidades adicionais de estratégias de marketing alimentar (Pires et al., 2018; Fernandes et al., 2017; Anderson et al., 2012).

Os polifenóis são compostos bioativos, geralmente envolvidos na defesa da planta contra a radiação ultravioleta e ataques de agentes patogénicos ou predadores (Dai e Mumper, 2010). Estes compostos podem distribuir-se de forma heterogénea por todas as partes da planta (madeira, casca, talos, vagens, folhas, frutas, raízes, flores, pólen e sementes), no entanto, a concentração em fitoquímicos tende a ser superior nas flores, uma vez que estas são o órgão reprodutor da planta (Hassan et al., 2015). Por esse motivo, a flor é uma parte importante da planta para a sua reprodução e para a propagação da espécie, e tratando-se de flores não tóxicas, estas podem ainda integrar a dieta alimentar, uma vez que contêm uma grande variedade de antioxidantes naturais (Zheng et al., 2018). Nas plantas edíveis, os polifenóis contribuem para a amargura, adstringência, cor, sabor, odor e estabilidade oxidativa (Zheng et al., 2018; Hassan et al., 2015). Por exemplo, alguns compostos fenólicos contribuem para o amargor e adstringência dos vegetais e frutas, causada essencialmente pela interação entre as proantocianidinas e as glicoproteínas presentes na saliva (Skrajad, 2017; Perez-Gregorio et al., 2014). As antocianinas, um dos seis subgrupos do grande grupo de componentes de polifenóis das plantas, conhecidas como flavonoides, são responsáveis pela coloração das mesmas, promovendo tonalidade que varia desde o amarelo, laranja, vermelho, azul até ao negro (Dabas, 2016). No entanto, a atual importância da composição fenólica de cada espécie vegetal prende-se com o seu contributo para a manutenção da saúde. Embora haja estudos que abordam a escassez de informação sobre a biodisponibilidade dos compostos fenólicos no metabolismo humano, muitos investigadores associam esses compostos bioativos aos seus benefícios, os quais incluem a capacidade potencial que estes antioxidantes têm para a eliminação de radicais livres, a atividade antimicrobiana, a prevenção de doenças cardiovasculares, o efeito protetor contra danos e doença hepáticos, as atividades antimutagénica e antineoplásica (Rasouli et al., 2017; Benvenuti et al., 2016; Dai e Mumper, 2010). 
Apesar das várias características supracitadas e do seu potencial agronómico, a ideia de comer flores ainda é vista com relutância. De facto, atualmente existe um notório tipo de neofobia, uma vez que, na maioria das vezes, um novo alimento cria uma desconfiança inata (Chen e Wei, 2017), especialmente em crianças (Dovey et al., 2008). Consequentemente, é necessário, em primeiro lugar, desenvolver a educação nutricional, avaliar as preferências do consumidor e introduzir as flores como alimento comum na população em geral. Por esses motivos, este trabalho experimental teve como objetivo estudar o teor de compostos não-nutrientes, nomeadamente, fenólicos totais, flavonoides totais e carotenoides totais, de diferentes flores cultivadas para consumo: calêndula (Calendula officinalis L.), camélia (Camellia japonica L.) e rosa (Rosa canina L.), bem como avaliar a atividade antioxidante das mesmas.

\section{MATERIAIS E MÉTODOS}

\subsection{Preparação das amostras}

Todas as pétalas estudadas (Calendula officinalis, Camellia japonica e Rosa canina) foram adquiridas já secas na empresa certificada de venda de produtos naturais online "Círculo Bio". As pétalas foram trituradas e homogeneizadas durante 30 a 60 segundos, utilizando um moinho Grindomix GM 200 (Retsch, Haan, Alemanha), a 2000 rpm. De seguida, foram acondicionadas em frascos de amostragem de plástico opaco, protegidas da luz e mantidas à temperatura ambiente até serem analisadas ( 2 semanas após receção).

\subsection{Determinação do teor de carotenoides totais}

Foram pesadas $5 \mathrm{~g}$ de cada amostra, adicionando-se-lhe $40 \mathrm{~mL}$ de acetona pura. Após agitação constante durante 15 minutos ao abrigo da luz, filtrou-se sob vácuo, adicionando-se, posteriormente ao filtrado $30 \mathrm{~mL}$ de éter de petróleo, com agitação. A solução resultante foi transferida para uma ampola de decantação, realizando-se uma lavagem com $3 \times 50 \mathrm{~mL}$ de água desionizada. A quantificação dos carotenoides, obtidos na fase orgânica, e cujo volume foi medido rigorosamente, foi realizada por espectrofotometria de UV/Vis, a um comprimento de onda de $450 \mathrm{~nm}$, usando éter de petróleo como branco, de acordo com a fórmula abaixo representada (1), sendo E1 o coeficiente de extinção molar com o valor de $2592 \mathrm{~mol}^{-1} \mathrm{~cm}^{-1} \mathrm{~L}$ (Almeida e Penteado, 1988).

$\mu \mathrm{g}$ de carotenoides/g amostra $=\frac{\text { Abs } \times \text { Volume } \times 10^{6}}{100 \times E 1^{\%} 1 \mathrm{~cm} \times \text { Peso da amostra }}$

\subsection{Preparação dos extratos}

A avaliação dos compostos bioativos de natureza hidrossolúvel (polifenóis totais e flavonoides totais) e da atividade antioxidante foi determinada em extratos hidroalcoólicos, utilizando-se como solvente uma solução água:etanol (50:50). Prepararam-se os extratos com $1 \mathrm{~g}$ de amostra e $50 \mathrm{~mL}$ de solvente. A extração foi efetuada em placa de aquecimento com agitação constante de $600 \mathrm{rpm}$ (Variomag, Telemodul $40 \mathrm{CT}$, Alemanha), durante 60 minutos, a $40{ }^{\circ} \mathrm{C}$. Comparando com outros estudos efetuados com diferentes matrizes, verifica-se que estas condições são das mais eficientes e sustentáveis para a obtenção de extratos, tal como 
descreve Costa etal. (2014). Seguidamente, os extratos obtidos foram filtrados e congelados a $-25{ }^{\circ} \mathrm{C}$, para posterior análise. Todos os extratos (três de cada amostra), referentes às espécies estudadas, foram realizados em triplicado.

\subsubsection{Determinação do teor de fenólicos totais}

Os compostos fenólicos totais dos extratos foram determinados pelo método espetrofotométrico, utilizando o reagente de Folin-Ciocalteu (RFC). Segundo metodologia previamente descrita por Vinha et al. (2015), colocaram-se num tubo de ensaio $500 \mu \mathrm{L}$ de extrato, branco ou padrão (solução de ácido gálico 1000 ppm), aos quais se adicionaram 2,5 $\mathrm{mL}$ de RFC diluído em água desionizada (1:10) e 2,0 mL de solução de carbonato de sódio $\left(\mathrm{Na}_{2} \mathrm{CO}_{3}\right) 7,5 \%(\mathrm{~m} / \mathrm{v})$. Os extratos foram mantidos a $45^{\circ} \mathrm{C}$, durante 15 minutos, ao abrigo da luz. Posteriormente, deixaram-se em repouso, à temperatura ambiente, durante 30 minutos. Foram efetuadas leituras das absorvências a $765 \mathrm{~nm}$ em leitor de microplacas (BioTek Synergy $H T$, GENS5, EUA). Os resultados são expressos em equivalentes de ácido gálico (EAG) em mg EAG/g de amostra.

\subsubsection{Determinação do teor de flavonoides totais}

A análise para a quantificação dos flavonoides totais seguiu a metodologia previamente descrita por Costa et al. (2014), com ligeiras modificações. A absorvência foi determinada a $510 \mathrm{~nm}$ e corresponde ao máximo de absorção do complexo $\mathrm{AlCl}_{3}$-flavonoide formado. $\mathrm{A}$ catequina (EC) foi o padrão utilizado para a construção da curva de calibração e respetiva quantificação dos flavonoides totais. Foram misturados rapidamente $1 \mathrm{~mL}$ de cada extrato, 4 $\mathrm{mL}$ de água desionizada e $300 \mu \mathrm{L}$ de nitrito de sódio a $5 \%(\mathrm{~m} / \mathrm{v})$. Após uma homogeneização de 5 minutos, adicionaram-se $300 \mu \mathrm{L}$ de $\mathrm{AlCl}_{3}$ a $10 \%(\mathrm{~m} / \mathrm{v})$. Posteriormente, adicionaram-se 2 $\mathrm{mL}$ de solução de hidróxido de sódio $(1 \mathrm{~mol} / \mathrm{L})$ e $2,4 \mathrm{~mL}$ de água desionizada. A mistura resultante foi homogeneizada em vórtex, imediatamente antes de proceder à leitura da absorvência num espectrofotómetro UV/Vis (Thermo, Genesys 10S UV-Vis, China). Os resultados, obtidos em triplicado, foram expressos em equivalentes de catequina ( $\mathrm{mg} \mathrm{EC/g}$ de amostra).

\subsection{Atividade antioxidante}

Os compostos antioxidantes podem ser definidos como substâncias que, quando presentes em pequenas concentrações em relação ao substrato oxidável, são capazes de retardar ou mesmo inibir substancialmente a oxidação do substrato (Niki, 2010).

\subsubsection{Inibição do radical livre DPPH•}

O método usado para a determinação da capacidade de neutralização do radical $\mathrm{DPPH}$ ' mede a captação deste radical através da diminuição da absorvência, medida a $525 \mathrm{~nm}$, que resulta da redução de um antioxidante $(\mathrm{AH})$ ou de uma reação com radicais livres. A metodologia consistiu em adicionar $20 \mu \mathrm{L}$ de cada extrato hidroalcoólico a $280 \mu \mathrm{L}$ de solução etanólica de $\mathrm{DPPH}^{\cdot}\left(6,0 \times 10^{-5} \mathrm{~mol} / \mathrm{L}\right)$, preparada no próprio dia, e efetuar as leituras a $525 \mathrm{~nm}$ em leitor de microplacas (BioTek Synergy HT, GENS5, EUA) (Vinha et al., 2015). O decréscimo de DPPH• foi determinado de 2 em 2 minutos, até a reação estabilizar, contabilizando-se 30 minutos no total. Os resultados são expressos em \% de inibição. A percentagem de inibição do radical 
$\mathrm{DPPH} \cdot$ foi calculada mediante a seguinte equação: \% I = [(ADPPH-As $\left.) / A_{D P P H}\right] \times 100$, sendo As a absorvência da solução amostra e ADPPH a absorvência da solução de DPPH•

\subsection{Análise estatística}

Para o tratamento e análise dos dados utilizou-se o software IBM ${ }^{\circ}$ SPSS Statistics versão 23.0 para Windows. Os resultados dos compostos bioativos foram apresentados na forma de média \pm desvio-padrão, por apresentarem comportamento aproximadamente normal (aproximadamente simétricas e com achatamento mesocúrtico). A diferença entre valores médios dos compostos fenólicos totais, flavonoides totais e carotenoides totais, e da atividade antioxidante nas três amostras, foi avaliada através de ANOVA e, após deteção de diferenças significativas, estas foram identificadas com o teste de comparação à posteriori de diferenças mínimas significativas (LSD). A relação entre concentração de fenólicos totais, flavonoides totais e carotenoides totais com a atividade antioxidante foi avaliada através do coeficiente de correlação de Spearman. Diferenças significativas foram definidas para $p<0,05$.

\section{RESULTADOS E DISCUSSÃO}

Os compostos bioativos analisados nas pétalas secas dos três géneros florais foram as substâncias fenólicas (fenólicos totais), flavonoides totais e carotenoides, cujos resultados estão apresentados na Tabela 1.

Tabela 1. Conteúdo em compostos bioativos contidos nas amostras de pétalas das flores edíveis

\begin{tabular}{|c|c|c|c|c|}
\hline & Calendula officinalis & Camellia japonica & Rosa canina & $p^{*}$ \\
\hline $\begin{array}{c}\text { Fenólicos totais } \\
\text { (mg EAG/g) }\end{array}$ & $13,7 \pm 0,1^{a}$ & $0,40 \pm 0,01^{\circ}$ & $1,86 \pm 0,02^{b}$ & $<0,001$ \\
\hline $\begin{array}{c}\text { Flavonoides totais } \\
(\mathrm{mg} \mathrm{EC} / \mathrm{g})\end{array}$ & $35,4 \pm 0,3^{b}$ & $35,0 \pm 0,5^{b}$ & $94,5 \pm 0,6^{a}$ & $<0,001$ \\
\hline $\begin{array}{c}\text { Carotenoides totais } \\
(\mu \mathrm{g} / \mathrm{g})\end{array}$ & $15,6 \pm 0,3^{a}$ & $0,16 \pm 0,02^{c}$ & $1,1 \pm 0,3^{b}$ & $<0,001$ \\
\hline
\end{tabular}

*ANOVA; ${ }^{a, b, c}$ Letras diferentes em cada linha/composto indicam diferenças significativas nas amostras de pétalas, de acordo com o teste de comparação à posteriori de diferenças mínimas significativas (LSD).

Através da análise dos resultados, verificam-se diferenças significativas entre as pétalas das três flores estudadas, no que diz respeito aos fenólicos totais, com variações entre 0,40 e 13,7 $\mathrm{mg} \mathrm{EAG/g}$. C. officinalis apresentou um elevado teor de fenólicos totais (13,7 mg EAG/g), comparativamente à $R$. canina e $C$. japonica (1,86 e 0,40 $\mathrm{mg}$ EAG/g, respetivamente). Em relação aos flavonoides totais, $R$. canina apresentou o maior conteúdo $(94,5 \mathrm{mg} \mathrm{EC} / \mathrm{g})$, enquanto $C$. japonica e $C$. officinalis apresentaram valores muito idênticos $(35,0 \mathrm{mg} \mathrm{EC} / \mathrm{g}$ e $35,4 \mathrm{mg} \mathrm{EC} / \mathrm{g}$, respetivamente). Quanto ao teor em carotenoides totais, também se observam grandes diferenças, detetadas como significativas $(p<0,05)$ entre as três amostras. Os resultados são apresentados em ordem decrescente: $C$. officinalis $(15,6 \mu \mathrm{g} / \mathrm{g})>R$. canina $(1,1$ $\mu \mathrm{g} / \mathrm{g})>C$. japonica $(0,16 \mu \mathrm{g} / \mathrm{g})$. Tendo em conta a tonalidade das pétalas da $C$. officinalis (laranja), era expectável que esta amostra apresentasse o maior teor em carotenoides.

Os resultados da análise da atividade antioxidante nas três flores encontram-se representados na Figura 1, expressos em \% de inibição do radical DPPH ${ }^{*}$. 


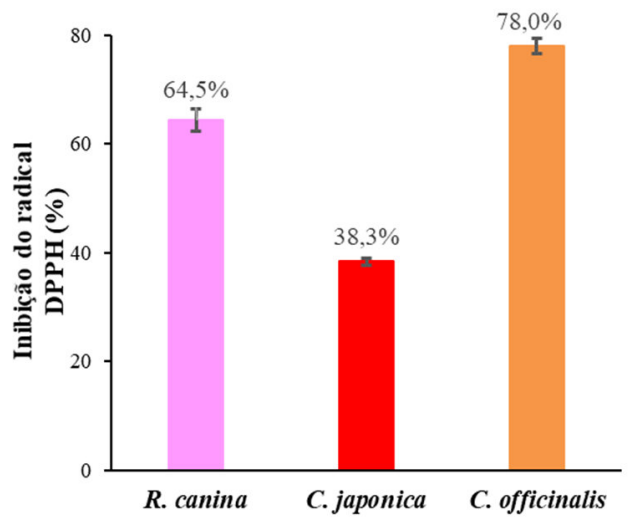

Figura 1. Atividade antioxidante (\% de inibição do radical DPPH•) média obtida nas três flores estudadas. Barra de erro corresponde ao desvio padrão.

De acordo com a Figura 1, a amostra que apresentou maior atividade antioxidante foi a $C$. officinalis (78,0\%), seguida da $R$. canina $(64,5 \%)$ e C. japonica $(38,3 \%)$ (ANOVA, $p<0,001$ ), respetivamente.

Tendo em conta que os compostos bioativos estudados contribuem para a atividade antioxidante, e dado que as três amostras não apresentaram um comportamento idêntico face ao conteúdo em compostos bioativos, foi estudada a correlação entre cada composto e a sua atividade antioxidante, através do coeficiente de correlação de Spearman (Tabela 2).

Tabela 2. Valores obtidos pela análise estatística da correlação ( $r S$, coeficiente de correlação de Spearman) entre os compostos bioativos e a atividade antioxidante (\% de inibição do radical DPPH•).

\begin{tabular}{lcc}
\cline { 2 - 3 } & rs & $\boldsymbol{p}$ \\
\hline Fenólicos totais vs. DPPH $^{\bullet}$ & 0,917 & 0,001 \\
Flavonoides totais vs. DPPH $^{\bullet}$ & 0,267 & 0,488 \\
Carotenoides totais vs. DPPH $^{\bullet}$ & 0,900 & 0,001 \\
\hline
\end{tabular}

Os fenólicos totais e os carotenoides totais revelaram uma correlação positiva muito boa com a atividade antioxidante $((r s=0,917(p=0,001)$ e $r s=0,900(p=0,001)$, respetivamente). Por outro lado, contrariamente ao expectável, os flavonoides totais não apresentaram correlação com a atividade antioxidante, com base nos valores de coeficiente de correlação baixos e não significativos $\left(\left(r_{s}=0,267(p=0,488)\right)\right.$. Estes resultados sugerem que, quanto maior o teor em fenólicos totais ou em carotenoides totais, maior a percentagem de inibição do DPPH, ou seja, maior a atividade antioxidante, conforme representa a Figura 2. 

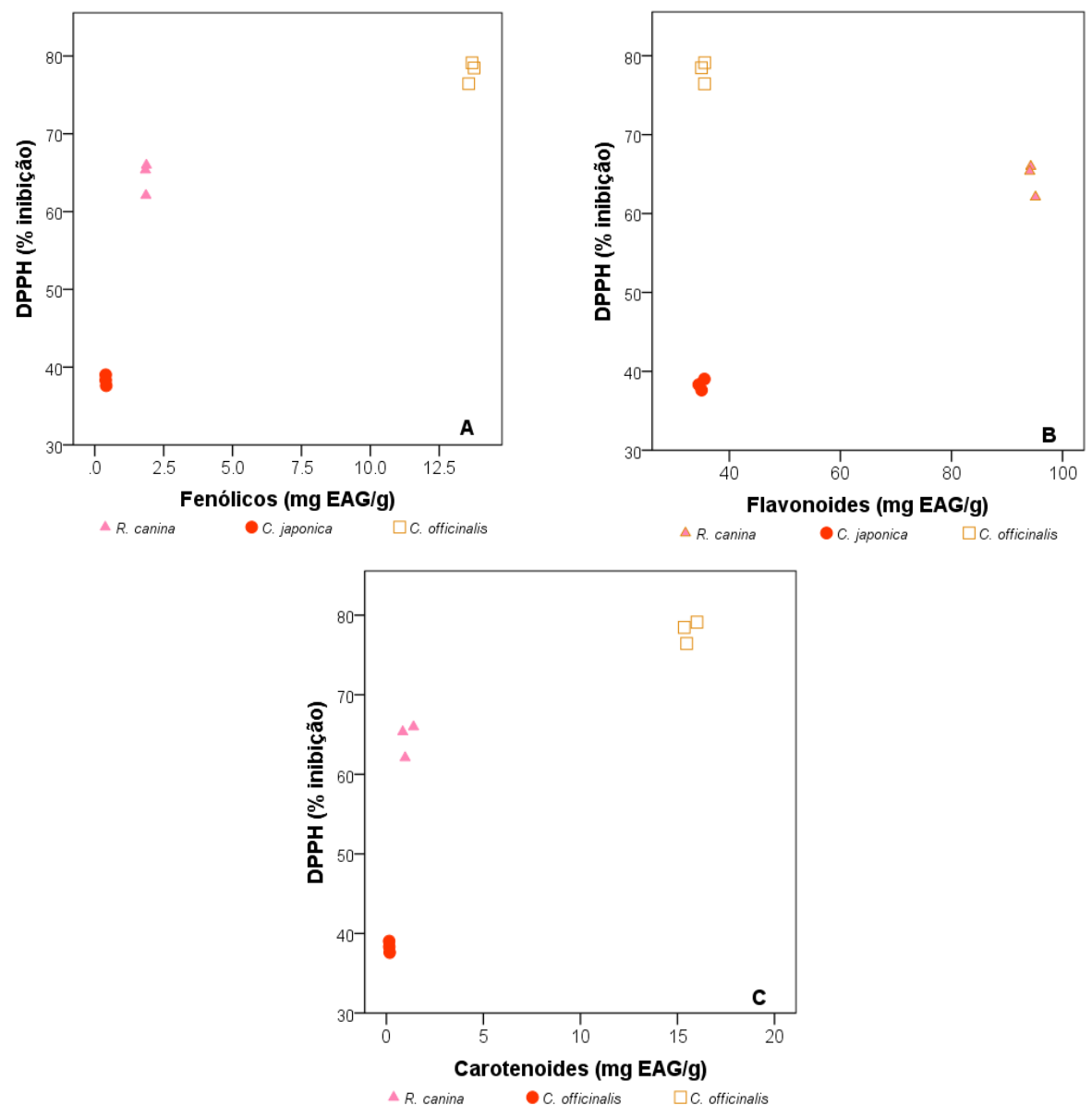

Figura 2. Correlação entre a atividade antioxidante (\% inibição do radical DPPH•) e os compostos fenólicos (A), flavonoides (B) e carotenoides (C).

Relativamente ao teor de compostos bioativos encontrados na $C$. officinalis, os resultados não estão de acordo com outros estudos publicados. Por exemplo, Velickovic et al. (2014), usando extratos hidroalcoólicos, obtiveram maiores teores de fenólicos totais e teores de flavonoides totais mais baixos $(29,79 \mathrm{mg} / \mathrm{g}$ e $0,17 \mathrm{mg} / \mathrm{g}$, respetivamente). A atividade antioxidante obtida foi superior $(96,85 \%)$ e, tal como no presente estudo, os autores encontraram uma forte correlação entre o conteúdo em fenólicos totais e a atividade antioxidante. Sabir et al. (2015) também relataram teores de fenólicos totais superiores e flavonoides totais mais baixos $(72,91$ $\mathrm{mg} / \mathrm{g}$ e $15,2 \mathrm{mg} / \mathrm{g}$, respetivamente), contudo estes valores dizem respeito a extratos aquosos. Quanto à atividade antioxidante, estes autores obtiveram percentagens de inibição do radical DPPH inferiores a $56 \%$ (em diferentes concentrações de DPPH). No presente estudo, C. officinalis apresentou uma atividade antioxidante superior $(78,0 \%)$. No caso de Petrova et al. (2016), os teores de fenólicos totais e flavonoides totais em extratos hidroalcoólicos das pétalas foram bastante inferiores (3,30 $\mathrm{mg} / \mathrm{g}$ e 1,40 $\mathrm{mg} / \mathrm{g}$, respetivamente). 
Em relação à $C$. japonica, os resultados obtidos, no que diz respeito à atividade antioxidante, estão em concordância com o estudo de Piao et al. (2011). A atividade antioxidante obtida pelos autores variou entre os $28 \%$ e $60 \%$, em diferentes concentrações de extrato etanólico. No presente estudo, C. japonica apresentou 38,3\% de atividade antioxidante. Por outro lado, num estudo realizado com diferentes variedades de $C$. japonica, Kanth et al. (2014) relataram teores de fenólicos totais entre 4,8 e 19,6 mg/g e teores de flavonoides totais entre 4,5 e 16,1 $\mathrm{mg} / \mathrm{g}$, de acordo com a cor das pétalas. Estes resultados, em comparação com o presente estudo, demonstram que o conteúdo em fenólicos totais é superior, enquanto o conteúdo em flavonoides totais é bastante inferior, facto que está diretamente associado à tonalidade das pétalas desta espécie botânica que pode variar entre branco e vermelho. As pétalas por nós estudadas apresentavam uma tonalidade rosa claro.

Quanto ao conteúdo em compostos bioativos presentes na $R$. canina, os resultados não estão de acordo com Barros et al. (2011). Estes autores apresentam resultados bastantes superiores aos encontrados neste estudo $(270,28 \mathrm{mg} / \mathrm{g})$, não acontecendo o mesmo em relação ao conteúdo de flavonoides totais, que foram mais baixos $(18,41 \mathrm{mg} / \mathrm{g})$. Estas diferenças podem ser explicadas pelo facto de terem usado extratos metanólicos das pétalas. Os carotenoides foram também estudados, contudo, apenas o licopeno e clorofilas foram quantificados, tendo os autores relatado elevadas concentrações dos mesmos (Barros et al., 2011).

Devido à escassez de estudos com pétalas de $R$. canina, os resultados experimentais foram também comparados com as folhas e frutos desta espécie. Em relação às folhas, Ghazghazi et al. (2010) relataram teores de fenólicos totais superiores $(5,42-9,21 \mathrm{mg} / \mathrm{g})$ e teores de flavonoides totais bastante inferiores $(0,11-0,44 \mathrm{mg} / \mathrm{g})$ às pétalas, tendo extraído o material vegetal seco com hexano, seguido de diclorometano e metanol. Quanto aos carotenoides, estes autores apenas quantificaram o licopeno e $\beta$-caroteno. Relativamente a extratos aquosos dos frutos, Demir et al. (2014) obtiveram um maior conteúdo de fenólicos totais $(31,08 \mathrm{mg} / \mathrm{g})$ e um teor mais baixo de flavonoides totais de soluções hidroalcoólicas $(9,48$ $\mathrm{mg} / \mathrm{g}$ ).

As diferenças observadas face ao teor de compostos bioativos entre o presente estudo e os estudos supracitados podem ser essencialmente devidas às condições edafo-climáticas e aos métodos analíticos adotados, nomeadamente, à escolha dos solventes para a preparação de extratos.

\section{CONCLUSÃO}

No presente trabalho experimental, foi reconhecida a presença de compostos bioativos e atividade antioxidante nas pétalas de $C$. officinalis, $C$. japonicae $R$. canina. De um modo geral, as determinações efetuadas demonstraram que $C$. officinalis apresentou o maior teor em fenólicos totais e carotenoides e, consequentemente, maior atividade antioxidante. Por outro lado, $R$. canina foi a espécie floral mais rica em flavonoides totais. C. japonica apresentou o menor conteúdo em todos os compostos bioativos estudados, assim como menor atividade antioxidante.

De acordo com estes resultados, os efeitos biológicos dos compostos bioativos quantificados neste estudo têm o potencial de promover a saúde, se as pétalas forem incorporadas diretamente na alimentação. É importante ter em conta que as propriedades dos compostos bioativos dependem da quantidade ingerida e da sua biodisponibilidade. Torna-se assim interessante mostrar o potencial destes géneros florais, na perspetiva de os integrar mais 
habitualmente na dieta alimentar, bem como em suplementos alimentares ou produtos farmacêuticos, sendo, no entanto, necessário realizar mais estudos.

\section{BIBLIOGRAFIA}

Almeida, LB, Penteado, MVC. (1988); "Carotenoids and pro-vitamin A value of white fleshed Brazilian sweet potatoes (Ipomoea batatas Lam.)". Journal of Composition and Analysis, 1(4): 249258. Doi: 10.1016/0889-1575(88)90034-8.

Anderson, JE, Goetz, CM, Mclaughlin, JL, Suffness, M. (2012); "A blind comparison of simple benchtop bioassays and human tumour cell cytotoxicities as antitumor prescreens". Phytochemical Analysis, 2(3): 107-111. Doi: 10.1002/pca.2800020303.

Bailão, EFLC, Devilla, IA, Conceição, EC, Borges, LL. (2015); "Bioactive compounds found in Brazilian Cerrado fruits". International Journal of Molecular Sciences, 16(10): 23760-23783. Doi: 10.3390/ijms161023760.

Barros, L, Carvalho, AM, Ferreira, ICFR. (2011); "Exotic fruits as a source of important phytochemicals: Improving the traditional use of Rosa canina fruits in Portugal". Food Research International, 44(7): 2233-2236. Doi: 10.1016/j.foodres.2010.10.005.

Benvenuti, S, Bortolotti, E, Maggini, R. (2016); "Antioxidant power, anthocyanin content and organoleptic performance of edible flowers". Scientia Horticulturae, 199: 170-177. Doi: 10.1016/j.scienta.2015.12.052.

Chen, N-H, Wei, S. (2017); "Factors influencing consumers' attitudes towards the consumption of edible flowers". Food Quality and Preference, 56 Part A: 93-100. Doi: 10.1016/j.foodqual.2016.10.001.

Ching, J, Soh, WL, Tan, C-H, Lee, J-F, Tan, J-YC, Yang, J, Yap, C-W, Koh, H-L. (2012); "Identification of active compounds from medicinal plants extracts using gas chromatography-mass spectrometry and multivariate data analysis". Journal of Separation Science, 35(1): 53-59. Doi: 10.1002/jssc.201100705.

Costa, ASG, Alves, RC, Vinha, AF, Barreira, SVP, Nunes, MA, Cunha, LM, Oliveira, MBPP. (2014); "Optimization of antioxidants extraction from coffee silverskin, a roasting by-product, having in view a sustainable process". Industrial Crops and Products, 53: 350-357. Doi: 10.1016/j.indcrop.2014.01.006.

Dabas, D. (2016); "Polyphenols as Colorants". Advances in Food Technology and Nutritional Sciences, SE(2): S1-S6. Doi: 10.17140/ AFTNSOJ-SE-2-101.

Dai, J, Mumper, RJ. (2010); "Plant phenolics: Extraction, analysis and their antioxidant and anticancer properties". Molecules, 15(10): 7313-7352. Doi: 10.3390/molecules15107313.

Demir, N, Yildiz, O, Alpaslan, M, Hayaloglu, AA. (2014); "Evaluation of volatiles, phenolic compounds and antioxidant activities of rose hip (Rosa L.) fruits in Turkey". LWT- Food Science and Technology, 57(1): 126-133. Doi: 10.1016/j.Iwt.2013.12.038.

Dovey, TM, Staples, PA, Gibson, EL, Halford, JC. (2008); “Food neophobia and 'picky/fussy' eating in children: a review". Appetite, 50(2-3): 181-193. Doi: 10.1016/j.appet.2007.09.009.

Fernandes, L, Casal, S, Pereira, JA, Saraiva, JA, Ramalhosa, E. (2017); "Edible flowers: A review of the nutritional, antioxidant, antimicrobial properties and effects on human health". Journal of Food Composition and Analysis, 60: 38-50. Doi: 10.1016/j.jfca.2017.03.017.

Ghazghazi, H, Miguel, GM, Hasnaoui, B, Sebei, H, Ksontini, M, Figueiredo, AC, Pedro, LG, Barroso, JG. (2010). "Phenols, essential oils and carotenoids of Rosa canina from Tunisia and their antioxidant activities". African Journal of Biotechnology, 9(18): 2709-2716. Disponível em: http://www.academicjournals.org/AJB. ISSN 1684-5315 @ 2010 Academic Journals. 
Guiné, RPF, Santos, E, Correia, PMR. (2017). "Edible Flowers: Knowledge and Consumption Habits". Acta Scientific Nutritional Health, 1(3): 18-22. Disponível em: https://www.researchgate.net/publication/318860985_EDIBLE_FLOWERS_KNOWLEDGE_AND_C ONSUMPTION_HABITS.

Hassan, IA, Nasiru, IA, Malut, AM, Abdulkadir, IS, Ali, AS. (2015); "Phytochemical studies and thin layer chromatography of leaves and flowers extracts of Senna siamea lam for possible biomedical applications". Journal of Pharmacognosy and Phythoterapy, 7(3): 18-26. Doi: 10.5897/JPP2014.0337.

Kanth, BK, Lee, KY, Lee, GJ. (2014); "Antioxidant and radical-scavenging activities of petal extracts of Camellia japonica ecotypes". Horticulture, Environment and Biotechnology, 55(4): 335-341. Doi: 10.1007/s13580-014-0024-7.

Kusuma, IW, Murdiyanto, Arung, ET, Syafrisal, Kim Y. (2014); "Antimicrobial and antioxidant properties of medicinal plants used by the Bentian tribe from Indonesia". Food Science and Human Wellness, 3(3-4): 191-196. Doi: 10.1016/j.fshw.2014.12.004.

Montes, CEM, Pacheco, SOS, Martínez, GA, Freitas, MR, Ivona, JG, Ivona, JA, Craig, WJ, Pacheco, FJ. (2018); "Long-Term Dietary Intake of Chia Seed Is Associated with Increased Bone Mineral Content and Improved Hepatic and Intestinal Morphology in Sprague-Dawley Rats". Nutrients, 10(7): pii E922. Doi: 10.3390/nu10070922.

Niki, E. (2010); "Assessment of antioxidant capacity in vitro and in vivo". Free Radical Biology and Medicine, 49(4): 503-515. Doi: 10.1016/j.freeradbiomed.2010.04.016.

Pereira, MC, Steffens, RS, Jablonski, A, Hertz, PF, Rios, AO, Vizzotto, M, Flôres, SH. (2013); "Characterization, bioactive compounds and antioxidant potential of three Brazilian fruits". Journal of Food Composition and Analysis, 29(1): 19-24. Doi: 10.1016/j.jfca.2012.07.013.

Perez-Gregorio, MR, Mateus, N, Freitas, V. (2014); "New procyanidin B3-human salivary protein complexes by mass spectrometry. Effect of salivary protein profile, tannin concentration, and time stability". Journal of Agricultural and Food Chemistry, 62(41): 10038-10045. Doi:10.1021/jf5033284.

Petrova, I, Petkova, N, Ivanov, I. (2016); "Five edible flowers - valuable source of antioxidants in human nutrition". International Journal of Pharmacognosy and Phytochemical Research, 8(4): 604610. Disponível em: www.ijppr.com. ISSN: 0975-4873.

Piao, MJ, Yoo, ES, Koh, YS, Kang, HK, Kim, J, Kim, YJ, Kang, HH, Hyun, JW. (2011); "Antioxidant effects of the ethanol extract from flower of Camellia japonica via scavenging of reactive oxygen species and induction of antioxidant enzymes". International Journal of Molecular Sciences, 12(4): 2618-2630. Doi: 10.3390/ijms12042618.

Pires, TCSP, Dias, MI, Barros, L, Calhelha, RC, Alves, MJ, Oliveira, MBPP, Santos-Buelga, C, Ferreira, ICFR. (2018); "Edible flowers as sources of phenolic compounds with bioactive potential". Food Research International, 105: 580-588. Doi: 10.1016/j.foodres.2017.11.014.

Rasouli, H, Farzaei, MH, Khodarahmi, R. (2017); "Polyphenols and their benefits: A review". International Journal of Food Properties, 20: 1700-1741. Doi: 10.1080/10942912.2017.1354017.

Rufino, MSM, Alves, RE, Fernandes, FAN, Brito, ES. (2011); "Free radical scavenging behavior of ten exotic tropical fruits extracts". Food Research International, 44(7): 2072-2075. Doi: 10.1016/j.foodres.2010.07.002.

Sabir, SM, Khan, MF, Rocha, JBT, Boligon, AA, Athayde, ML. (2015); "Phenolic profile, antioxidant activities and genotoxic evaluations of Calendula officinalis". Journal of Food Biochemistry, 39(3): 316-324. Doi: $10.1111 /$ jfbc.12132.

Shukla, A, Tyagi, R, Vats, S, Shukla, RK. (2016); "Total phenolic content, antioxidant activity and phytochemical screening of hydroalcoholic extract of Casearia tomentosa leaves". Journal of Chemical and Pharmaceutical Research, 8(1): 136-141. Disponível em: www.jocpr.com/articles/total-phenolic-content-antioxidant-activity-and-phytochemicalscreening-of-hydroalcoholic-extract-of-casearia-tomentosa.pdf. 
Skrajad, MN. (2017); "Phenolic compounds and antioxidant activity of edible flowers". Journal of Education, Health and Sport, 7(8): 946-956. Doi: 10.5281/zenodo.995637.

Swithinbank, A. (2015); “Top 10 edible flowers”. Kitchen Garden, 7: 68-69.

Tundis, R, Marrelli, M, Conforti, F, Tenuta, MC, Bonesi, M, Menichini, F, Loizzo, MR. (2015); "Trifolium pratenseand T. repens (Leguminosae): Edible flower extracts as functional ingredients". Foods, 4(3): 338-348. Doi: 10.3390/foods4030338.

Velicković, JM, Dimitrijević, DS, Mitić, SS, Mitić, MN, Kostić, DA. (2014); "The determination of phenolics composition, antioxidant activity and heavy metals in the extracts of Calendula officinalis L". Advanced Technologies, 3(2): 46-51. Disponível em: http://www.tf.ni.ac.rs/casopisarhiva/sveska3vol2/c6.pdf.

Vinha, AF, Guido, LF, Costa, ASG, Alves, RC, Oliveira, MBPP. (2015); "Monomeric and oligomeric flavan-3-ols and antioxidant activity of leaves from different Laurus $s p$ ". Food \& Function, 6(6): 1944-1949. Doi: 10.1039/c5fo00229j.

Vinha, AF, Alves, RC, Barreira, SVP, Castro, A, Costa, ASG, Oliveira, MBPP. (2014); "Effect of peel and seed removal on the nutritional value and antioxidant activity of tomato (Lycopersicon esculentum L.) fruits". LWT - Food Science and Tecnology, 55(1): 197-202. Doi: 10.1016/j.Iwt.2013.07.016.

Virgolin, LB, Seixas, FRF, Janzantti, NS. (2017); "Composition, content of bioactive compounds, and antioxidant activity of fruit pulps from the Brazilian Amazon biome". Pesquisa Agropecuária Brasileira, 52(10): 933-941. Doi: 10.1590/S0100-204X2017001000013.

Xu, Y, Fan, M, Ran, J, Zhang, T, Sun, H, Dong, M, Zhang, Z, Zheng, H. (2016); "Variation in phenolic compounds and antioxidant activity in apple seeds of seven cultivars". Saudi Journal of Biological Sciences, 23(3): 379-388. Doi: 10.1016/j.sjbs.2015.04.002.

Zheng, J, Yu, X, Maninder, M, Xu, B. (2018); "Total phenolics and antioxidants profiles of commonly consumed edible flowers in China". International Journal of Food Properties, 21(1): 1524-1540. Doi: 10.1080/10942912.2018.1494195. 
